\title{
REVIEW
}

\section{Airway Management: Methods of Oxygenation}

\author{
Ryan Decoste $\mathrm{BScH}^{1}$, Nada Ismaiel MSc BScH${ }^{1}$, David Burnett $\mathrm{BSc}^{2}$, Michael Wong MD BScH${ }^{2}$, \\ Orlando Hung MD FRCPC ${ }^{3}$
}

${ }^{1}$ Class of 2015, Faculty of Medicine, Dalhousie University
${ }^{2}$ Class of 2014, Faculty of Medicine, Dalhousie University
${ }^{3}$ Department of Anesthesia, Dalhousie University

Abstract

Airway management in the emergency or elective setting encompasses a collection of important skills and training necessary for all health care providers. The purpose of this review is to provide trainees with a general overview of the important techniques and strategies used in airway management. We present a case scenario of a failed airway to illustrate the clinical application of these techniques. Special attention is given to bag-mask-ventilation, the use of extra-glottic devices, tracheal intubation, and surgical airways, as these are the basic airway management techniques.

Furthermore, we describe factors that influence the selection of an airway management technique depending on the situation, including the training and skills of the individual practitioner, the availability of equipment and medications, the level of acuity (elective vs. emergency), as well as patient-specific factors. The goal is to equip trainees with basic knowledge surrounding emergency airway management and encourage trainees to seek clinical opportunities to practice these skills.

\section{Case Presentation}

A 41-year old male with a large body habitus (BMI 35 $\mathrm{kg} / \mathrm{m} 2$ ) is scheduled for cystoscopy and insertion of a ureteric stent for renal stones at a local tertiary care center. He has no known allergies and general anesthesia was previously well-tolerated. His past medical history is significant only for an uncomplicated laparoscopic cholecystectomy two years ago. He is otherwise well and does not take any medications. He has been NPO for 12 hours prior to his procedure and his history does not suggest significant anesthetic risk.

Upon arrival in the Operating Room (OR), all routine monitors (ECG monitor, pulse oximeter, and noninvasive blood pressure) were placed on the patient. Intravenous access was secured in the left antecubital fossa. Following denitrogenation, general anesthesia was induced with a combination of midazolam (Versed), fentanyl (Sublimaze), and propofol (Diprivan). Immediately following induction, the patient vomited into the mask, aspirated, and his oxygenation quickly desaturated to $40 \%$. How should this patient's airway be managed and what interventions are necessary?

\section{Introduction}

A patient's inability to ventilate and oxygenate is always a medical emergency. Urgent measures must be taken to restore tissue oxygenation to prevent serious damage to major organs (e.g. brain) or death. The primary goal of emergency airway management is to restore oxygenation, with a secondary goal of protecting the airway from aspiration of foreign substances. To accomplish these goals, the practitioner must have a series of back-up plans available in case of a failed airway, defined as three unsuccessful attempts at direct laryngoscopy or the inability to maintain acceptable tissue oxygenation. ${ }^{1}$ In recent years several airway management algorithms, including the American Society of Anesthesiologists (ASA) Difficult Airway Management Algorithm, have been developed to guide practitioners towards effective evidence-based methods to restore oxygenation. ${ }^{2}$ Unfortunately, algorithms have limitations and must be used as informative guides depending on the clinical context, rather than as rigid instructions.

While restoring and maintaining oxygenation in the event of an emergency is crucial, practitioners should note that in the event of a cardiac arrest, Cardiopulmonary Resuscitation (CPR) by chest compressions should take priority over definitive airway management by intubation. Given that bag-mask ventilation (BMV) is a key aspect of CPR, a strong BMV technique is important when CPR takes priority over tracheal intubation.

Significant innovation has led to the development of new techniques that aid practitioners in managing 
emergency, difficult or failed airways, including the introduction of lightwand devices, video, and fiberoptic technologies. Examples of airway management modalities include BMV, extraglottic devices (EGDs), tracheal intubation (TI) and surgical airway techniques. Despite a significant focus on TI in clinical training and airway management literature, practitioners should ideally be skilled in other techniques of oxygenation as well. The ultimate goal is to achieve adequate oxygenation by whatever means necessary to prevent hypoxemia, which is associated with significant morbidity and mortality. ${ }^{1}$

The goal of this review is to provide a brief overview of various airway management techniques to help the reader appreciate strategies to manage difficult airways. These techniques will be discussed in the context of a failed airway case. The information herein is not an exhaustive review of airway management devices and techniques. The interested reader can find further information in more detailed resources. ${ }^{1,3-6}$

\section{Bag-Mask-Ventilation}

BMV is an important airway management skill to master for all airway practitioners. BMV is routinely used in the operating room (OR), emergency department, and pre-hospital settings. The necessary equipment for BMV is widely available, portable, and relatively inexpensive. BMV is a non-invasive method

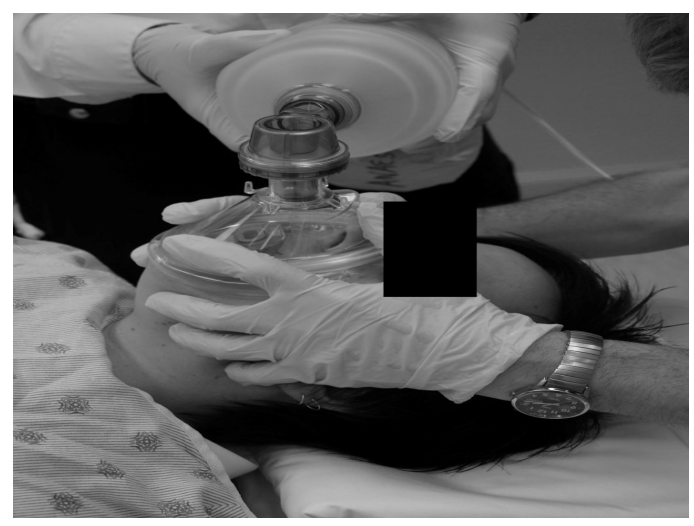

Figure 1A: Bag-Mask ventilation: Two-person technique

to manage the compromised airway, especially in the event of a failed intubation. ${ }^{7,8}$

To perform BMV, the skilled practitioner should create and maintain a tight seal on the facemask and ensure the upper airway is patent by placing the patient's head and neck into a "sniffing position" with a chin-lift or jaw thrust. ${ }^{9,10}$ This maximizes airway opening and prevents the tongue and epiglottis from obstructing the glottis. The stability of the cervical spine should be assessed prior to manipulating the head and neck, especially in trauma settings. The mask should be held continuously to the patient's face using a single hand to create a tight mask seal while squeezing the bag with the other hand to provide ventilation (Figure 1B). ${ }^{9}$ Maintaining a tight seal, elevating the tongue and epiglottis with a chin lift or head tilt, as well as squeezing the ventilation bag by a single-operator during BMV can be challenging. An oral or nasal airway can be used to aid in maintaining an open airway by preventing the tongue from obstructing the glottis. ${ }^{8}$ If mask seal is difficult, a two-person maneuver is ideal, as a tight seal can be better maintained between the face and mask with two hands by the practitioner while another practitioner squeezes the bag for ventilation (Figure 1A). Although this is optimal, it is not always practical, especially in the emergency setting where resources may be limited. A single rescuer may be required to manually ventilate a patient for an extended period of time, so practitioners must become proficient in performing BMV individually.

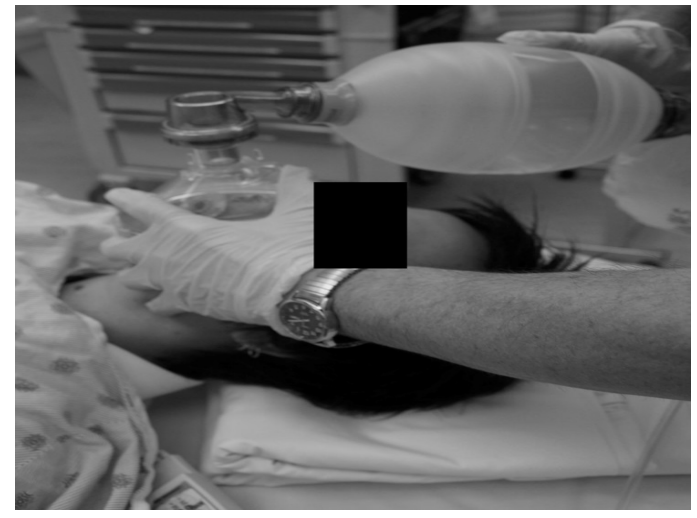

Figure 1B: Bag-Mask ventilation: One person technique

Several anatomical features can render BMV difficult or even futile, which can be remembered using the mnemonic 'BOOTS': Bearded, Older, Obese, Toothless, Stridor/Snoring. ${ }^{11-13}$ Additionally, patients with anatomic features that warrant a higher Mallampati grade (Grade III or IV) may present difficulty in performing BMV (Figure 2). ${ }^{8}$ Over-inflation of the lungs must be avoided during BMV, as this can cause barotrauma and gastric distension. ${ }^{14}$ 


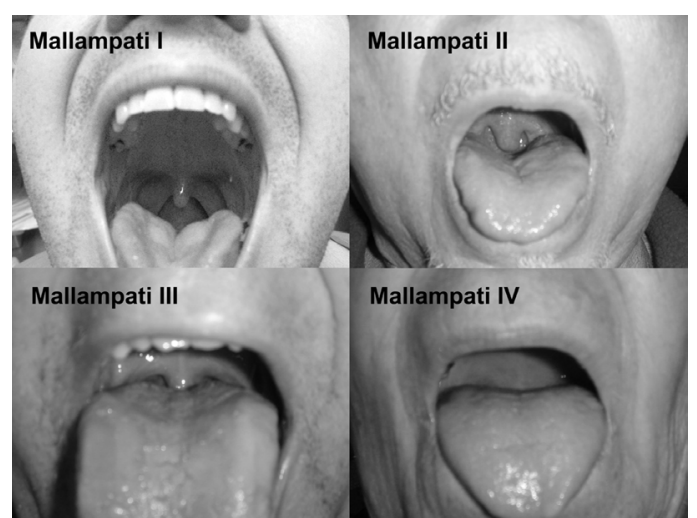

Figure 2: Mallampatti Classification Score

\section{Extraglottic Devices}

EGDs comprise a variety of devices that direct airflow into the glottic inlet while sealing the periglottic area

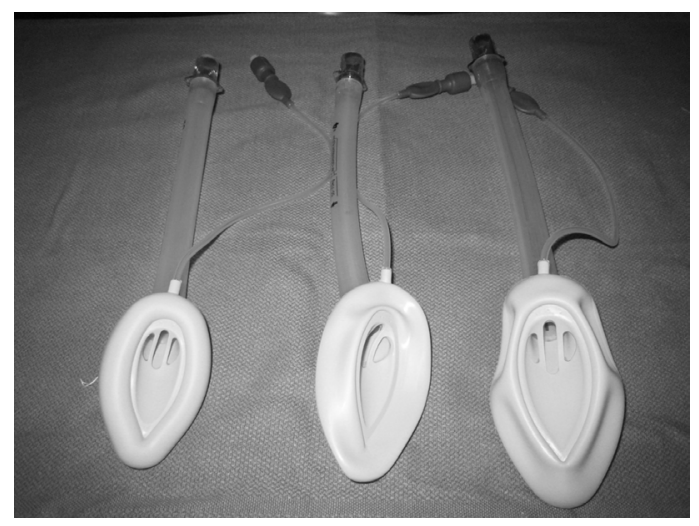

Figure 3: Laryngeal Mask Airway (LMA) Classic in various sizes.

with an inflatable cuff or balloon. EGDs are known for their relative ease of use compared to TI. ${ }^{15,16}$ Properly placed EGDs can quickly restore oxygenation in the emergency setting and reduce complications resulting from prolonged hypoxia. EGDs have been used successfully to provide ventilation in patients with characteristics of difficult tracheal intubation, and some EGDs have been developed to facilitate TI. ${ }^{17}$ Significant disadvantages of EGDs include: difficult placement in patients with small mouth opening or distorted upper airway anatomy; reduced protection against gastric aspiration, although rare compared to $\mathrm{TI} ;{ }^{17-20}$ and potential for impaired local tissue perfusion with over-inflation of sealing cuffs, causing ischemic injury to the airway mucosa. ${ }^{17,21}$
The use of EGDs for proper airway management is largely supported in the literature. Their ease of use and versatility make EGDs popular among airway management practitioners in different clinical settings, and they are recommended in the ASA and other difficult airway algorithms as a primary rescue device or planned backup maneuver during a failed airway.,22 The most common EGD used in clinical practice is the Laryngeal Mask Airway (LMA) Classic (LMA North America Inc., San Diego, CA), which is popular for its ease of use and effectiveness in establishing an airway during both routine and emergency situations (Figure 3). For proper placement, the distal end of the device cuff is placed into the hypopharynx just above the upper esophageal sphincter and inflated. Suboptimal placement carries a risk of gastric insufflation and/or aspiration.17 LMA should be avoided in obstetrical patients, and patients with a history of hiatal hernia, gastroesophageal reflux, or bowel obstruction as serious or fatal gastric aspirations associated with the use of LMA have been reported..$^{23-26}$

The LMA has largely replaced TI in many anesthetized patients undergoing simple surgical procedures with low risk of aspiration and has been incorporated into CPR protocols, transportation of critically ill patients and ICU settings. In addition, its utility as a rescue device for a difficult or failed airway cannot be overlooked. In this setting, the LMA should only be used as a temporizing measure due to risk of aspiration and inability to remove pulmonary secretions. ${ }^{17} \mathrm{~A}$ newer EGD, the LMA Proseal (LMA North America Inc., San Diego, CA), combines a better seal with a drainage tube as an esophageal conduit to minimize the risk of gastric insufflation and aspiration. The conduit permits insertion of a gastric tube up to size 18 French. ${ }^{27}$ Numerous other EGDs are available on the market, such as the Combitube and King Laryngeal Tube. In-depth discussion of these specific products is beyond the scope of this overview. ${ }^{17}$

\section{Tracheal Intubation}

Several important attributes make TI the standard of care in airway management. The strength of the tracheal tube (TT) and the distance it extends into the trachea create a "patent" airway - one that is free from compromise following loss of consciousness or narrowing of the airway due to edema or compression. ${ }^{3}$ $\mathrm{TI}$ also offers a safe pulmonary route of administration for several drugs if intravenous access is not possible. ${ }^{6}$ 
While specific indications for TI vary widely, ${ }^{5,6}$ general indications for TI include: loss of autonomous airway

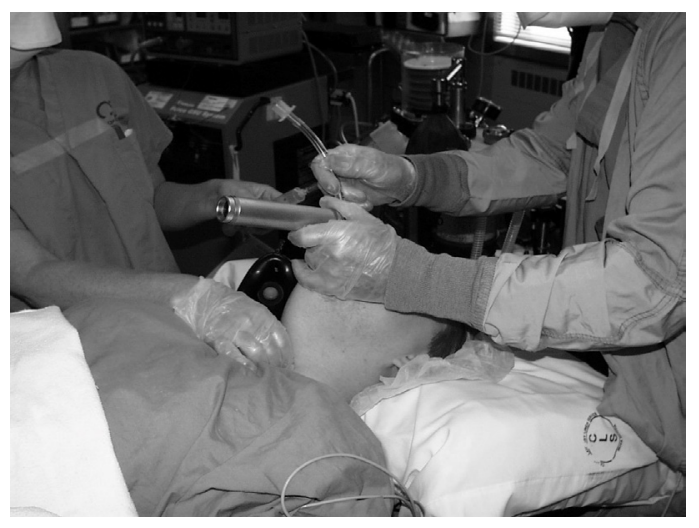

Figure 4: Tracheal intubation technique under direct laryngoscopy using a Macintosh laryngoscope.

maintenance; failure of ventilation with other airway devices; or loss of consciousness when undergoing general anesthesia. TI entails inserting a TT through the mouth or nose into the upper airway past the epiglottis and vocal cords, and into the trachea. Classically, TI is achieved under direct laryngoscopy - a technique requiring considerable training and dexterity (Figure 4). A thorough discussion of laryngoscopy equipment and techniques can be found in other resources. ${ }^{1}$

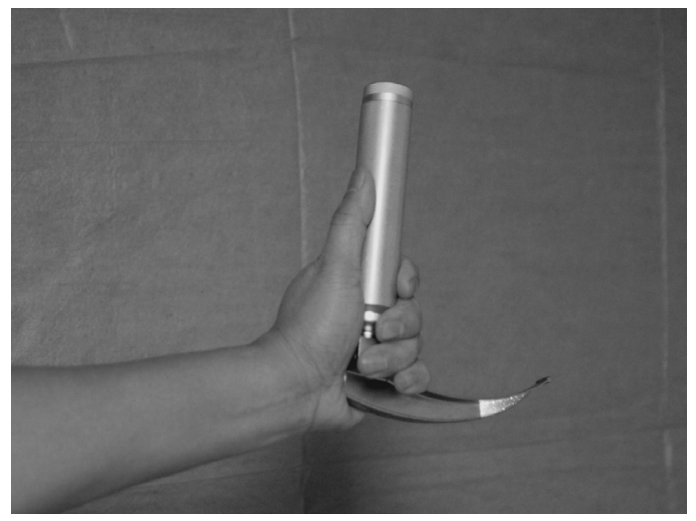

Figure 5: Macintosh laryngoscope with proper grip

\section{Visual Laryngoscopy}

To achieve a direct line-of-sight from the maxillary teeth to the larynx, the patient's head must be tilted back into the "sniffing" position (as with BMV). Positioning for intubation can be further optimized by placing rolled towels, a pillow, or another supporting structure under the patient's shoulders to maximize neck extension and improve direct visualization of the epiglottis and vocal cords. Furthermore, the bed should be lowered or raised to best suit the practitioner's height as this will improve visualization and manipulation of equipment relative to the patient's position on the bed.

These techniques may enhance the chance of a successful intubation on the first attempt. Medications for sedation and muscle relaxation are required to facilitate laryngoscopy in elective intubation. To perform laryngoscopy, the curved blade of the Macintosh laryngoscope (Figure 5) is advanced through the mouth with the left hand, with the tip of the blade placed into vallecula to indirectly elevate the epiglottis by applying pressure to the hyoepiglottic ligament. The laryngoscope is lifted away from the patient's face while avoiding the use of maxillary teeth as a fulcrum. This maneuver aligns the axes of the mouth, pharynx, and larynx, and establishes a direct line-of-sight to the vocal cords. ${ }^{28}$

In the event of a prolonged or difficult intubation during which medications for sedation and muscle relaxation have been administered without successful intubation, apneic oxygenation may be considered. With apneic oxygenation, high-flow (12-15 L/min) oxygen can be delivered through nasal prongs despite the lack of ventilation at the present time. While this idea is relatively novel, it has been shown to improve overall oxygen saturation, especially in obese patients in whom intubation can be technically more challenging. ${ }^{29}$

Intubation is guided by a light source at the distal end of the laryngoscope blade, allowing the TT to be passed through the vocal cords and into the trachea using the right hand (Figure 4). A syringe is used to inflate air into the cuff on the distal end of the tube to seal the trachea and prevent aspiration. An oxygen delivery system is attached to the proximal end of the tube, and tube placement is confirmed by monitoring end-tidal CO2. Disposable colorimetric end-tidal CO2 detectors may be connected between the TT and bag in emergency situations outside the OR. To avoid tube placement into the right main-stem bronchus, it is necessary to auscultate the lungs for equal breath sounds bilaterally. ${ }^{28,30}$

Repeated attempts at TI can traumatize the upper airway, causing tissue edema and hindering further attempts at intubation. Therefore, it is critical to optimize the chance of successful intubation with each 
attempt using new strategies and accessories. With each failed attempt at TI, practitioners should consider changing the laryngoscope, changing the blade/size on the laryngoscope, introducing any of the accessories discussed below, or even allowing a nearby trained practitioner to attempt the TI. Several innovative airway management accessories have been developed to aide in visualizing the vocal cords. While direct laryngoscopy is often successful with a conventional Macintosh laryngoscope, anticipated difficult intubations may warrant the use of video laryngoscopy (e.g. GlideScope ${ }^{\circ}$ ) for better visualization of the glottis. Skill is required, however, as insertion of the TT may be more difficult than with direct visualization. More information about video laryngoscopy can be found in other resources. ${ }^{31}$

Numerous tools and accessories can increase the chance of a successful intubation, including the tracheal introducer (commonly known as "gum elastic bougie"), flexible fiberoptic bronchoscopy, and rigid and semirigid fiberoptic stylets. The gum elastic bougie serves as a sturdy shaft over which the TT is guided into the trachea. Similarly, a TT is passed over a flexible bronchoscope that is guided into the trachea by mechanical controls, with the added benefit of indirect visualization of the airway through a lens. ${ }^{1}$

\section{Non-Visual Laryngoscopy}

Lightwands represent an innovative technique of intubation without visualization of the glottis. All lightwands consist of a rigid or semirigid shaft with a distal light source. There are several brands of lightwands, including the popular Trachlight ${ }^{\circ}$, which includes a retractable wire stylet that is removed prior to entering the trachea.

The advantage of the lightwand is the use of transillumination of anterior neck soft tissues to confirm its presence in the trachea, allowing for intubation without direct visualization and with minimal mouth opening. The light appears as a clearly demarcated red circle located slightly below the thyroid prominence when the tip of the TT with the lightwand inside is in the glottic opening. The light appears diffuse if the tip is in the esophagus. Although lightwand-guided intubation without a laryngoscope requires additional training, ${ }^{32}$ this technique can be an important option when direct laryngoscopy is impossible due to presence of blood and secretions, cervical spine immobility, or anatomic variation. ${ }^{6}$ Their low cost and relative ease of use make the lightwand a popular and effective tool in urgent situations.

\section{Surgical Airways}

Surgical airway techniques are essential for practitioners responsible for airway management. These life-saving procedures are reserved for situations where all other airway management techniques have failed to provide oxygenation. In these situations, in the hands of non-surgical clinicians (anesthesiologists, emergency physicians and paramedics), cricothyrotomy is preferred over tracheotomy because it is easy to perform with minimal equipment and has fewer complications. ${ }^{33,34}$

Difficult surgical airways can be anticipated using the 'SHORT' acronym (Surgery or fixed cervical Spine flexion deformity, Hematoma, Obesity, prior Radiation to the neck, and Laryngotracheal Tumor). Potential complications of surgical airways include: misplacement (i.e. through the thyrohyoid membrane above the larynx); long execution time; failed tube placement; and significant hemorrhage. ${ }^{34,35}$ Long-term complications include: subglottic stenosis; dysphonia; injury to the recurrent laryngeal nerve; pulmonary aspiration; fracture of tracheal cartilage and esophageal perforation. ${ }^{36}$ However, failure to perform a surgical airway as a last resort results in failure to oxygenate the patient and ultimately death. Serious complications leading to long-term morbidity are rare and outweighed by the benefits of establishing oxygenation. Thus, there are no absolute contraindications to performing an emergency surgical airway. ${ }^{34}$ Brief overviews of

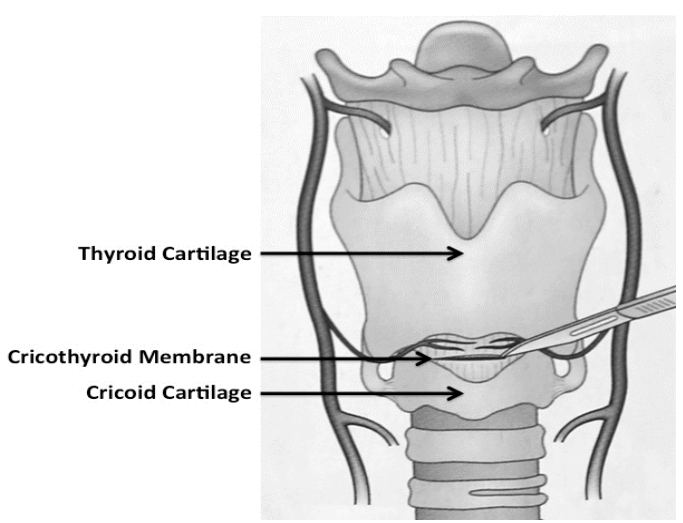

Figure 6: Cricothyrotomy incision in the cricothyroid membrane.

common surgical airway techniques are presented below. More detailed descriptions of each procedure can be found in Hung and Murphy, 2011. 1,34 


\section{Open Cricothyrotomy}

Open cricothyrotomy is a tactile, rather than visual, technique. All efforts to locate surface landmarks of the cricothyroid membrane (CTM) should be performed prior to initiating the procedure. To locate the CTM, palpate along the anterior neck from the mandible down to find the first firm prominence (thyroid cartilage), and then palpate more caudally to find the second firm prominence (cricoid cartilage). The space between the thyroid and cricoid cartilages is the CTM. ${ }^{37}$ The CTM is actually more cephalad than its assumed position by many practitioners, and thus more care should be invested into properly locating the CTM. Once identified, a $4 \mathrm{~cm}$ vertical midline incision is made over the CTM, followed by a transverse incision of the CTM at the superior border of the cricoid cartilage (Figure 6). Inferior retraction should be performed with a tracheal hook, followed by insertion of a dilator (or the index finger) and finally, a cuffed TT or tracheotomy tube. Placement should be confirmed with end-tidal $\mathrm{CO}_{2}$ and auscultation immediately followed by $x$-ray to confirm adequate positioning and rule out lung injury and pneumothorax. Since this is a rescue airway technique, cricothyrotomy should be converted to a tracheotomy once the patient is stable. ${ }^{34}$

\section{Seldinger Cricothyrotomy}

Many practitioners are more comfortable performing

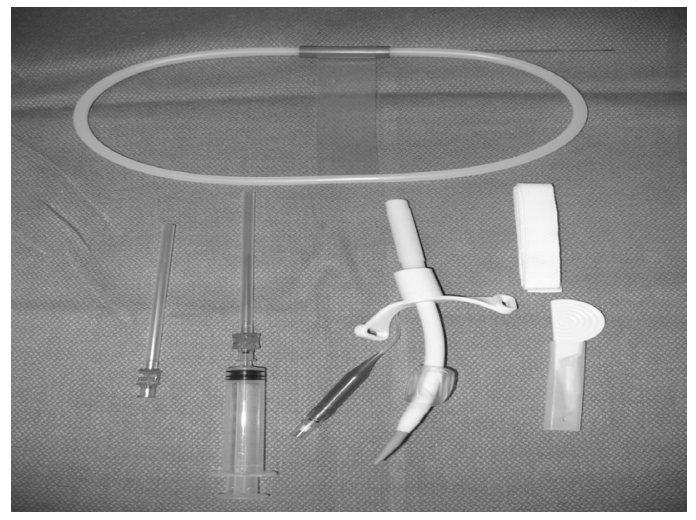

Figure 7: Seldinger cricothyrotomy kit.

Seldinger cricothyrotomy compared to open cricothyrotomy. In this procedure, a vertical stab incision is made in the midline through the skin overlying the CTM. An 18-gauge needle with a catheter attached to a fluid-filled syringe (Figure 7) is inserted caudally through the CTM. Placement in the trachea is confirmed by aspirating air, after which the needle and syringe are removed to leave the catheter in place. A guide-wire is inserted through the catheter before its removal. A small incision is made along the guide-wire. Finally, a cuffed airway loaded onto a dilator is inserted through this incision over the guide-wire. The guide-wire and dilator are then removed. Once stable, a definitive tracheotomy should be performed. ${ }^{34}$

\section{Case Discussion}

In this case, immediately after the patient vomited and aspirated, the patient's mouth was suctioned to remove regurgitated gastric contents. Ideally, tracheal intubation should be performed to allow immediate pulmonary toileting. Unfortunately, the anesthesiologist was planning to use an LMA Classic for oxygenation during the surgical procedure and tracheal intubation was not prepared. In view of the severe hypoxemia, BMV was performed with $100 \%$ oxygen to improve oxygenation. Oxygenation only improved to $80 \%$ with BMV. After preparing the patient for tracheal intubation with the administration of Suxamethonium chloride (Succinylcholine) for muscle relaxation, direct laryngoscopy was attempted unsuccessfully due to poor visualization of the glottis. Video-laryngoscopy using the GlideScope ${ }^{\circledast}$ was attempted. Although visualization of the glottis was improved, TI remained unsuccessful due to the inability to advance the TT through the glottis. A flexible bronchoscope was subsequently used to guide the TT into the trachea. However, in the presence of blood, secretions, and edema secondary to multiple intubation attempts, visualization of the glottis using the bronchoscope was difficult. Since oxygenation deteriorated to 50\%, an LMA was promptly inserted and oxygenation quickly improved to $82 \%$. In view of the multiple failed intubation attempts and the suboptimal oxygen saturation with the LMA, an otolaryngologist was consulted to perform a surgical tracheotomy to allow bronchoscopy and pulmonary toileting and to improve oxygenation. Although a successful tracheotomy was placed, it required three attempts. Bronchoscopy and pulmonary toileting were performed immediately after tracheotomy and the oxygen saturation gradually improved to $94 \%$. The surgical procedure was cancelled and the patient was transferred to the ICU in stable condition for further management and monitoring.

This case demonstrates a range of airway management strategies that may be employed in an emergency situation. In such situations, preparation is crucial and it is always necessary to consider accessible back-up plans prior to any airway management procedure. Proper planning allows for reduced airway trauma by avoiding repeated attempts at a single method, 
and could prove life-saving in the event of an airway emergency.

\section{Summary}

Airway management is all about oxygenation and not just tracheal intubation. The literature demonstrates that persisting with failing airway techniques rather than defaulting to alternative rescue strategies results in higher rates of morbidity and mortality. Practitioners should be skilled in all techniques of oxygenation. In fact, having a planned strategy improves the success of airway rescue and reduces the incidence of adverse outcomes, including death. ${ }^{1}$

\section{References}

1. Hung OR, Murphy MF. Management of the difficult and failed airway. 2nd ed. New York: McGraw-Hill Professional, 2011.

2. Apfelbaum JL, Hagberg CA, Caplan RA, Blitt CD, Connis RT, Nickinovich DG et al. Practice guidelines for management of the difficult airway: An updated report by the american society of anesthesiologists task force on management of the difficult airway. Anesthesiology 2013;118(2):251-270.

3. Benumof J, Hagberg CA. Benumof's airway management: Principles and practice. 2nd ed. Philadelphia: Elsevier Health Science, 2007.

4. Roberts JR, Hedges JR, eds. Clinical procedures in emergency medicine. 4th ed. Philadelphia: Saunders, 2004.

5. Walls RM, Murphy MF, eds. Manual of emergency airway management. 4th ed. Lippincott Williams \& Wilkins, 2012.

6. Orebaugh SL. Atlas of airway management: Tools and techniques. 1st ed. Philadelphia: Lippincott Williams \& Wilkins, 2007.

7. Stockinger ZT, McSwain NE Jr. Prehospital endotracheal intubation for trauma does not improve survival over bag-valvemask ventilation. J Trauma 2004;56(3):531-536.

8. Kovacs G, Murphy MF. Manual noninvasive ventilation: Bagmask-ventilation. In: Hung OR, Murphy MF, eds. Management of the difficult and failed airway. 2nd ed. New York: McGraw-Hill Professional, 2012:113.

9. Lee HY, Jeung KW, Lee BK, Lee SJ, Jung YH, Lee GS et al. The performances of standard and ResMed masks during bag-valvemask ventilation. Prehosp Emerg Care 2013;17(2):235-240.

10. Matioc AA. Training novice users in bag-valve-mask technique. Prehosp Disaster Med 2010;25(4):370-371.

11. Yildiz TS, Solak M, Toker K. The incidence and risk factors of difficult mask ventilation. J Anesth 2005;19(1):7-11. Yildiz TS, Solak M, Toker K. The incidence and risk factors of difficult mask ventilation. J Anesth 2005;19(1):7-11.

12. Langeron O, Masso E, Huraux C, Guggiari M, Bianchi A, Coriat $\mathrm{P}$ et al. Prediction of difficult mask ventilation. Anesthesiology 2000;92(5):1229-1236.

13. Kheterpal S, Han R, Tremper KK, Shanks A, Tair AR, O'Reilly M et al. Incidence and predictors of difficult and impossible mask ventilation. Anesthesiology 2006;105(5):885-891.

14. Wenzel V, Idris AH, Banner MJ, Kubilis PS, Williams JL Jr. Influence of tidal volume on the distribution of gas between the lungs and stomach in the nonintubated patient receiving positivepressure ventilation. Crit Care Med 1998;26(2):364-368.

15. Mulcaster JT, Mills J, Hung OR, MacQuarrie K, Law JA, Pytka $\mathrm{S}$ et al. Laryngoscopic intubation: Learning and performance. Anesthesiology 2003;98(1):23-27.

16. Weksler N, Tarnopolski A, Klein M, Schily M, Rozentsveig V, Shapira AR et al. Insertion of the endotracheal tube, laryngeal mask airway and oesophageal-tracheal combitube. A 6-month comparative prospective study of acquisition and retention skills by medical students. Eur J Anaesthesiol 2005;22(5):337-340.

17. Hinkewich C, Hung OR, Coonan TJ. Extraglottic devices for ventilation and oxygenation. In: Hung OR, Murphy MF, eds. Management of the difficult and failed airway. 2nd ed. New York: McGraw-Hill Professional, 2012:201.

18. Boyle MJ, Flavell E. Which is more effective for ventilation in the prehospital setting during cardiopulmonary resuscitation, the laryngeal mask airway or the bag-valve-mask? - A review of the literature. Journal of Emergency Primary Health Care 2010;8(3):1.

19. Brimacombe JR, Berry A. The incidence of aspiration associated with the laryngeal mask airway: A meta-analysis of published literature. J Clin Anesth 1995;7(4):297-305.

20. Yu SH, Beirne OR. Laryngeal mask airways have a lower risk of airway complications compared with endotracheal intubation: A systematic review. J Oral Maxillofac Surg 2010;68(10):2359-2376.

21. Martins RH, Braz JR, Defaveri J, Gregorio EA, Abud TM. Effect of high laryngeal mask airway intracuff pressure on the laryngopharyngeal mucosa of dogs. Laryngoscope 2000;110(4):645-650.

22. Murphy MF, Crosby ET. The algorithms. In: Hung OR, Murphy MF, eds. Management of the difficult and failed airway. 2nd ed. New York: McGraw-Hill Professional, 2012:15.

23. Nanji GM, Maltby JR. Vomiting and aspiration pneumonitis with the laryngeal mask airway. Can J Anaesth 1992;39(1):69-70.

24. Ismail-Zade IA, Vanner RG. Regurgitation and aspiration of gastric contents in a child during general anaesthesia using the laryngeal mask airway. Paediatr Anaesth 1996;6(4):325-328.

25. Griffin RM, Hatcher IS. Aspiration pneumonia and the laryngeal mask airway. Anaesthesia 1990;45(12):1039-1040.

26. Keller C, Brimacombe J, Bittersohl J, Lirk P, von Goedecke A. Aspiration and the laryngeal mask airway: Three cases and a review of the literature. Br J Anaesth 2004;93(4):579-582.

27. Keller C, Brimacombe J. Mucosal pressure and oropharyngeal leak pressure with the ProSeal versus laryngeal mask airway in anaesthetized paralysed patients. Br J Anaesth 2000;85(2):262266.

28. Calder I, Picard J, Chapman M, O'Sullivan C, Crockard HA. Mouth opening: A new angle. Anesthesiology 2003;99(4):799-801.

29. Ramachandran SK, Cosnowski A, Shanks A, Turner CR. Apneic oxygenation during prolonged laryngoscopy in obese patients: a randomized, controlled trial of nasal oxygen administration. J Clin Anes 2010;22:164-168.

30. Levitan RM, Kovacs G. Direct laryngoscopy. In: Hung OR, Murphy MF, eds. Management of the difficult and failed airway. 2nd ed. New York: McGraw-Hill Professional, 2012:124.

31. Cooper RM, Law JA. Rigid and semirigid fiberoptic and video laryngoscopy and intubation. In: Hung OR, Murphy MF, eds. Management of the difficult and failed airway. 2nd ed. New York: McGraw-Hill Professional, 2012:159.

32. Christodoulou CC, Hung OR. Nonvisual intubation techniques. In: Hung OR, Murphy MF, eds. Management of the difficult and failed airway. 2nd ed. New York: McGraw-Hill Professional, 2012:186.

33. Ger R, Evans JT. Tracheostomy: An anatomico-clinical review. Clinical Anatomy 1993;6(6):337.

34. Launcelott GO, Johnson LB. Surgical airway. In: Hung OR, Murphy MF, eds. Management of the difficult and failed airway. 2nd ed. New York: McGraw-Hill Professional, 2012:222.

35. Erlandson MJ, Clinton JE, Ruiz E, Cohen J. Cricothyrotomy in the emergency department revisited. J Emerg Med 1989;7(2):115-118.

36. Boon JM, Abrahams PH, Meiring JH, Welch T. Cricothyroidotomy: A clinical anatomy review. Clinical Anatomy 2004;17(6):478-486.

37. McGill J, Clinton JE, Ruiz E. Cricothyrotomy in the emergency department. Ann Emerg Med 1982;11(7):361-364 